\title{
Origin of the Local Bubble
}

\author{
Burkhard Fuchs · Dieter Breitschwerdt • \\ Miguel A. de Avillez • Christian Dettbarn
}

Received: 15 February 2008 / Accepted: 5 August 2008 / Published online: 3 September 2008

(C) Springer Science+Business Media B.V. 2008

\begin{abstract}
We present a new unbiased search for OB associations in the Solar neighbourhood which have hosted the progenitor stars of the core collapse supernovae responsible for the Local Bubble in the interstellar gas. For this purpose we have analyzed a volume complete set (with a diameter of $400 \mathrm{pc}$ ) of B stars drawn from the Hipparcos catalogue and the Arivel data base, from which candidate members were selected by a kinematical criterion. After careful dereddening the star colours we have constructed a colour-magnitude diagram and confirmed that the Upper Scorpius, Upper Centaurus Lupus, and Lower Centaurus Crux subgroups of the Sco OB2 association are the youngest nearby OB associations. We dated their ages with theoretical isochrones in the range of 20-30 Myr, in agreement with previous work. We have traced backwards in time the paths of the stars and found that they entered the volume of the present bubble at 10 to $15 \mathrm{Myr}$ ago. We argue that the Local Bubble began to form then and estimate that 14 to 20 supernovae have exploded since. The implied energy input into the ambient medium can be shown to be sufficient to excavate a bubble of the presently observed size.
\end{abstract}

Keywords Open clusters and associations: Sco OB2 - ISM: local bubble

\section{Introduction}

The Sun is located inside the Local Bubble (LB) in the interstellar medium (ISM), a low density X-ray emitting cavity which is deficient of HI. Sfeir et al. (1999) and Lallement et

\footnotetext{
B. Fuchs $(\bowtie) \cdot$ C. Dettbarn

Astronomisches Rechen-Institut am Zentrum für Astronomie der Universität Heidelberg, Mönchhofstraße 12-14, 69120 Heidelberg, Germany

e-mail: fuchs@ari.uni-heidelberg.de

D. Breitschwerdt

Institut für Astronomie der Universität Wien, Türkenschanzstraße 17, 1180 Wien, Austria

e-mail: breitschwerdt@astro.univie.ac.at
}

M.A. de Avillez

Department of Mathematics, University of Evora, R. Romao Ramalho 59, 7000 Evora, Portugal 
al. (2003) have delineated the outer boundaries of the LB by studying NaI absorption lines of the ISM in the spectra of stars for which trigonometric parallaxes obtained in the Hipparcos mission (ESA 1997) were available. The outer walls of the LB show up as a sudden increase of the equivalent widths of the NaI lines, when the lines of sight towards these stars are passing through the walls. According to these measurements the LB extends to about $200 \mathrm{pc}$ in the Galactic plane, and $600 \mathrm{pc}$ perpendicular to it, but is inclined about 20 degrees relative to the direction of the Galactic pole. Thus the LB has an orientation perpendicular to Gould's Belt.

The physical status of the ISM within the LB has been observed in X-rays and by EUV spectroscopy. However, the interpretation of the observations is controversial, all the more the X-ray observations are complicated by charge exchange reactions between solar wind ions (SWCE) and heliospheric gas (Lallement 2004). We do not go into any details here but refer to the accompanying paper by Breitschwerdt et al. (2008).

The LB is situated next to the Loop I superbubble, from which it is separated by a wall showing up in absorption of soft X-rays in ROSAT PSPC images (Egger and Aschenbach 1995).

It has been suggested early that the LB has been excavated by core collapse supernovae (Cox and Anderson 1982; Innes and Hartquist 1984; Smith and Cox 2001) even though the LB does not contain any $\mathrm{OB}$ associations which might have harboured the progenitors of the SNII.

The search for the "smoking gun" of the origin of the LB proved partially successful by discovering that moving groups of young stars in the solar neighbourhood could provide an adequate number of SN explosions while crossing the path of the LB. Berghöfer and Breitschwerdt (2002, henceforth BB02) calculated the trajectory of the Pleiades subgroup B1 backwards in time, and found that 19 SNe could have exploded between 10-20 Myr ago in the region that is occupied by the LB. The remaining stars of B1 are now part of the Scorpius Centaurus OB association. It could be shown that this is in good agreement with the size of the LB and the present soft X-ray emissivity. A similar analysis was carried out by Maíz-Apellániz (2001), who calculated backwards in time the trajectories of Sco Cen subgroups and claimed that about $6 \mathrm{SNe}$ that went off in the Lower Centaurus Crux subgroup of the Sco OB2 association 7 to $9 \mathrm{Myr}$ ago formed the LB.

However, the search for possible OB associations was not very systematical, mainly because their discovery history is unclear. Thus Fuchs et al. (2006) were prompted to carry out a fresh and unprejudiced search and scrutinized all stars that are within a volume of about $400 \mathrm{pc}$ in diameter centered around the Sun, and performed a selection according to spectral and kinematical properties. The latter is based on three dimensional space velocities of the stars. Thus our approach is complementary to studies like by de Zeeuw et al. (1999) which are based on proper motions alone. Sartori et al. (2003) did include radial velocities when analyzing the subgroups of the Sco OB2 association, but work from a list of stars preselected by de Zeeuw et al. (1999). From the positions of B stars in the HR diagram and the main sequence's turnoff point we could reliably determine the age of the associations and the number of SNe within the volume occupied at present by the Local Bubble.

\section{Search for Nearby OB Associations}

Fuchs et al. (2006) tried to improve and harmonize previous studies. As a starting point we used the Hipparcos catalogue (ESA 1997) from which we selected all stars bluer than $(B-V)<-0.05$ with parallaxes larger than 5 milliarcseconds, because otherwise the distances would be to inaccurate. Drimmel et al. (2000) find by a comparison with the Tycho 
catalogue that the Hipparcos catalogue is about 97 percent complete down to $V=7.5$ which corresponds at a distance of $1 /(5$ mas $)=200 \mathrm{pc}$ to an absolute magnitude of $M_{\mathrm{V}}=1$. Allowing for an extinction of $A_{\mathrm{V}} \leq 0.2$ we have $M_{\mathrm{V}}<1.2$, and we have chosen the colour cut in $(B-V)$ accordingly (cf. Fig. 4). We have obtained in this way an unbiased complete sample of 762 B stars within a distance of 200 pc from the Sun. All stars in our list appear in the Arivel data base, which combines Hipparcos parallaxes and proper motions with radial velocity data collected from the literature (Wielen et al., in preparation). We find that for 610 stars radial velocities are available. These are accurate enough to be used for a kinematical analysis (cf. Fig. 2). We have tested if the availability of radial velocities is correlated with the colours of the stars and find that the 152 out of 762 stars for which no radial velocity is known are redder than $(B-V)>-0.1$. Fuchs et al. (2006) could show that this does not have any significant effect on the expected number of supernovae. For each star, spatial $X, Y$, and $Z$ coordinates and the corresponding $U, V$, and $W$ velocity components have been calculated. The coordinates are centered on the Sun with $X$ pointing towards $l=0^{\circ}$, $b=0^{\circ}, Y$ towards $l=90^{\circ}, b=0^{\circ}$, and $Z$ towards $b=90^{\circ}$, respectively. Since OB associations disperse slowly on time scales of the order of several $10^{7}$ years (Blaauw 1964), we have searched for kinematically coherent structures in our sample. For this purpose we have traced the paths of the stars backward in time over $3 \times 10^{7}$ years. Stellar orbits have been calculated using the epicyclic equations of motion for the stars in the sample (Lindblad 1959).

$$
\begin{aligned}
X(t)= & X(0)-\frac{V(0)}{-2 B}(1-\cos (\kappa t))+\frac{U(0)}{\kappa} \sin (\kappa t), \\
U(t)= & U(0) \cos (\kappa t)-\frac{\kappa}{-2 B} V(0) \sin (\kappa t), \\
Y(t)= & Y(0)+2 A\left(X(0)-\frac{V(0)}{-2 B}\right) t \\
& +\frac{\Omega_{0}}{-B \kappa} V(0) \sin (\kappa t)+\frac{2 \Omega_{0}}{\kappa^{2}} U(0)(1-\cos (\kappa t)), \\
V(t)= & \frac{-2 B}{\kappa} U(0) \sin (\kappa t)+V(0) \cos (\kappa t), \\
Z(t)= & \frac{W(0)}{v} \sin (v t)+Z(0) \cos (v t), \\
W(t)= & W(0) \cos (v t)-Z(0) v \sin (v t) .
\end{aligned}
$$

Here $\kappa$ denotes the epicyclic frequency, $\kappa=\sqrt{-4 \Omega_{0} B} . A$ and $B$ are the Oort constants, and $\Omega_{0}$ is the angular frequency of the rotation of the local standard of rest around the Galactic centre, $\Omega_{0}=V_{\mathrm{LSR}} / R_{\odot} \cdot v$ denotes the vertical oscillation frequency which is related to the local density $\rho_{0}$ by the Poisson equation as $v=\sqrt{4 \pi G \rho_{0}}$, where $G$ is the constant of gravitation. For the angular velocity of the local standard of rest we have adopted a value of $\Omega_{0}=220 \mathrm{~km} / \mathrm{s} / 8 \mathrm{kpc}$. The Oort constants describe in (1) the smooth Galactic gravitational potential, which is consistent with an essentially flat shape of the local Galactic rotation curve, $A=-B=\Omega_{0} / 2$ (Feast and Whitelock 1997). For the local density we adopt a value of $\rho_{0}=0.1 \mathscr{M}_{\odot} / \mathrm{pc}^{3}$ (Holmberg and Flynn 2004). These parameter values imply $\kappa=0.039 \mathrm{~km} / \mathrm{s} / \mathrm{pc}=4 \times 10^{-8} \mathrm{yr}^{-1}$ and $v=0.074 \mathrm{~km} / \mathrm{s} / \mathrm{pc}=7.5 \times 10^{-8} \mathrm{yr}^{-1}$. In Fig. 1 we show the positions of the stars today and $3 \times 10^{7}$ years ago. Most of the 610 stars do not belong to the $\mathrm{OB}$ association, which hosted the SNe responsible for the origin of the 

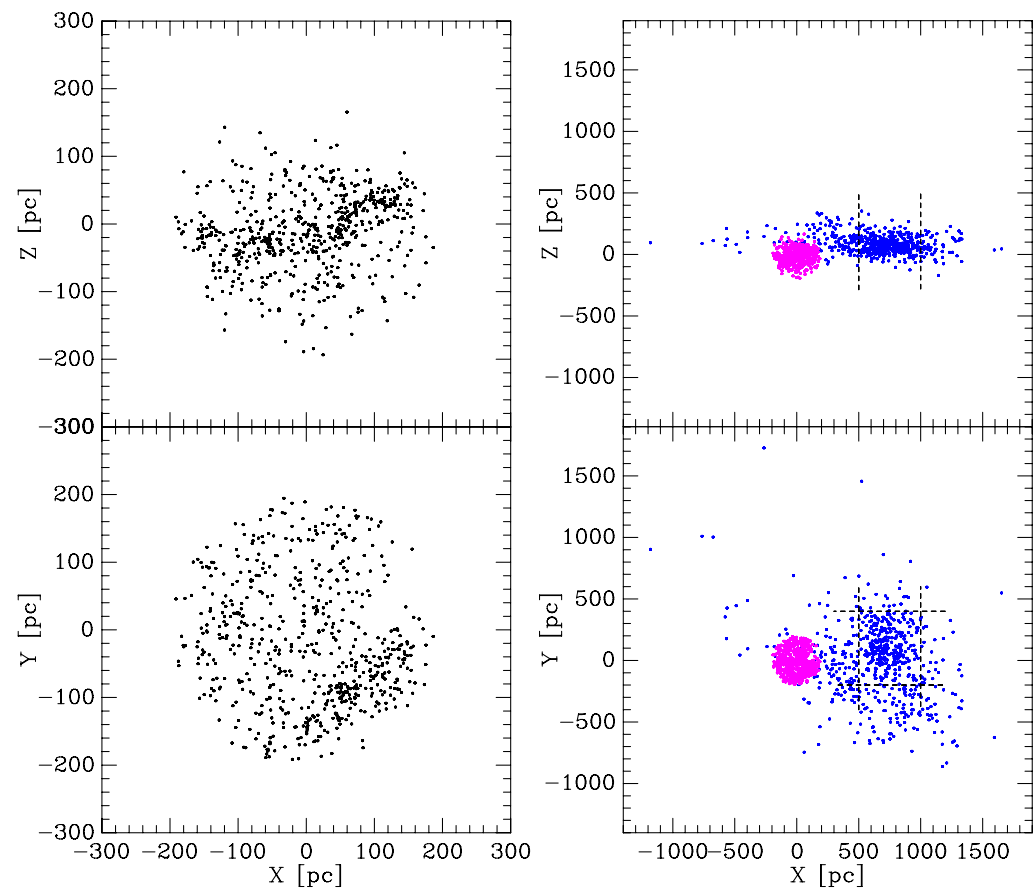

Fig. 1 Left panel: Positions of 610 stars drawn from the Hipparcos catalogue. The selected stars have colours $B-V<-0.05$ and for each star its radial velocity is known. The $X$-axis points towards the Galactic centre, $Y$ into the direction of Galactic rotation, and $Z$ towards the Galactic north-pole, respectively. Right panel: Positions of the originally selected stars today (pink) and $3 \times 10^{7}$ years ago (blue). The Sun is at rest in the diagrams. Stars lying in the windows indicated by dashed lines are identified as putative members of the searched for $\mathrm{OB}$ association

$\mathrm{LB}$, and have space velocities different from the velocity of the association. Thus they are dispersed away into a wide cloud. However, the overdense regions in Fig. 1 indicate that there is a considerable number of stars which stayed together. The larger size of the overdense regions in the back projected sample compared to its size today is obviously due to the observational errors. The typical accuracy of Hipparcos proper motions is about 1 mas/yr which corresponds at a distance of $100 \mathrm{pc}$ to a velocity of $0.5 \mathrm{~km} / \mathrm{s}$, whereas the accuracy of the radial velocities is several $\mathrm{km} / \mathrm{s}$, dominating the errors of the resulting space velocities. Assuming for the latter in each component an error of $5 \mathrm{~km} / \mathrm{s}$ (cf. Fig. 2) we expect errors in the star positions $3 \times 10^{7}$ years ago of about $150 \mathrm{pc}$ in each direction. Thus, if the overdensity of stars representing the kinematically homogeneous group is today occupying an area in $X$ and $Y$ of $200 \times 200 \mathrm{pc}$ (cf. Fig. 2), it will have been artificially spread out to a size of roughly $500 \times 500 \mathrm{pc}$ in $X$ and $Y$. As can be seen from Fig. 1 there is an outer shroud of stars which lies at greater distances from the core of the overdensity. These must be stars with genuinely different space velocities from the kinematically homogenous group of stars. We identify this kinematically homogeneous group of stars as an OB association and select 302 stars lying in the windows indicated as dashed lines in Fig. 1. As expected these stars are more or less closely related to the Sco OB2 association.

In Fig. 2 we show the present day velocity distribution of the 302 selected stars. Since the velocity dispersion of an OB association is of the order of $10 \mathrm{~km} / \mathrm{s}$ (Blaauw 1964) or even less (Kamaya 2004), we make a second selection indicated by windows drawn as dashed 

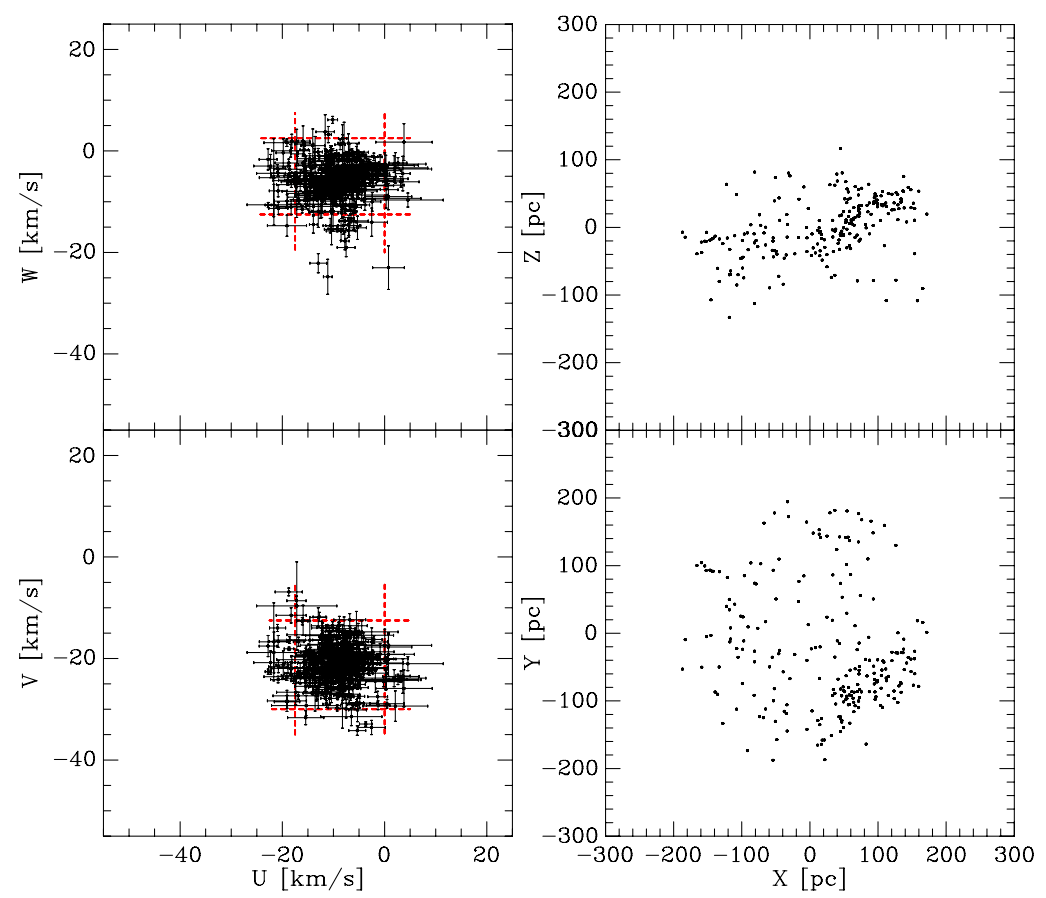

Fig. 2 Left panel: Present day velocity distribution of the 302 selected stars. A second selection is made of the stars lying in the windows indicated by dashed lines. Right panel: Spatial distribution of the finally 236 selected stars

lines in Fig. 2. This leaves a sample of 236 stars which we analyze in the following. Their spatial distribution is shown in the right panel of Fig. 2 where the Sco OB2 association is now clearly discernible. In Fig. 3 we reproduce a colour image of parts of the association.

The final sample is shown as a colour-magnitude diagram in Fig. 4. For this purpose we had to cross-identify the sample stars in the Geneva photometry data base (Mermilliod et al. 2000) and replaced the $(B-V)_{T}$ colours given in the Hipparcos catalogue by $(B-V)_{J}$ colours, because they can be then directly compared with theoretical isochrones available in the literature. In the colour range, which we consider here, $B-V$ given in the Tycho system can not be transformed directly to the Johnson system (ESA 1997). The absolute magnitudes have been determined from the visual magnitudes given in the Hipparcos catalogue in the Johnson system.

We have compared our sample with the extensive membership list of the Sco OB2 association compiled by de Zeeuw et al. (1999) who applied a combination of a modified convergent point method and the so called spaghetti method (Hoogerwerf and Aguilar 1999) to Hipparcos data. Of particular interest turned out the membership lists of the subgroups Upper Scorpius (US), Upper Centaurus Lupus (UCL), and Lower Centaurus Crux (LCC). With only very few exceptions all stars in the membership lists, which fulfill our colour selection criterion, appear also in our sample, which gives confidence in our selection procedure. A few stars from our final sample could be identified additionally in the membership list of de Geus et al. (1989) as members of the subgroups. The 79 stars common to both lists are colour coded in the right panel of Fig. 4. Of these stars 10 are belonging to the US, 42 to the UCL, and 27 stars belong to the LCC subgroup. 


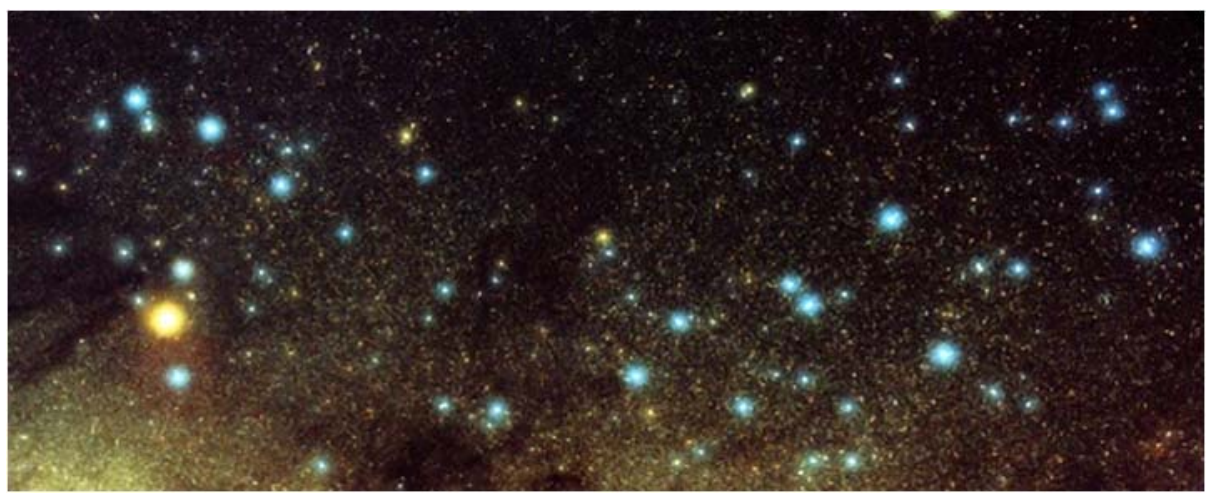

Fig. 3 Optical image of the Upper Scorpius (left) and Upper Centaurus Lupus (right) subgroup of the Sco OB2 association (copyright: T. Preibisch, Bonn)
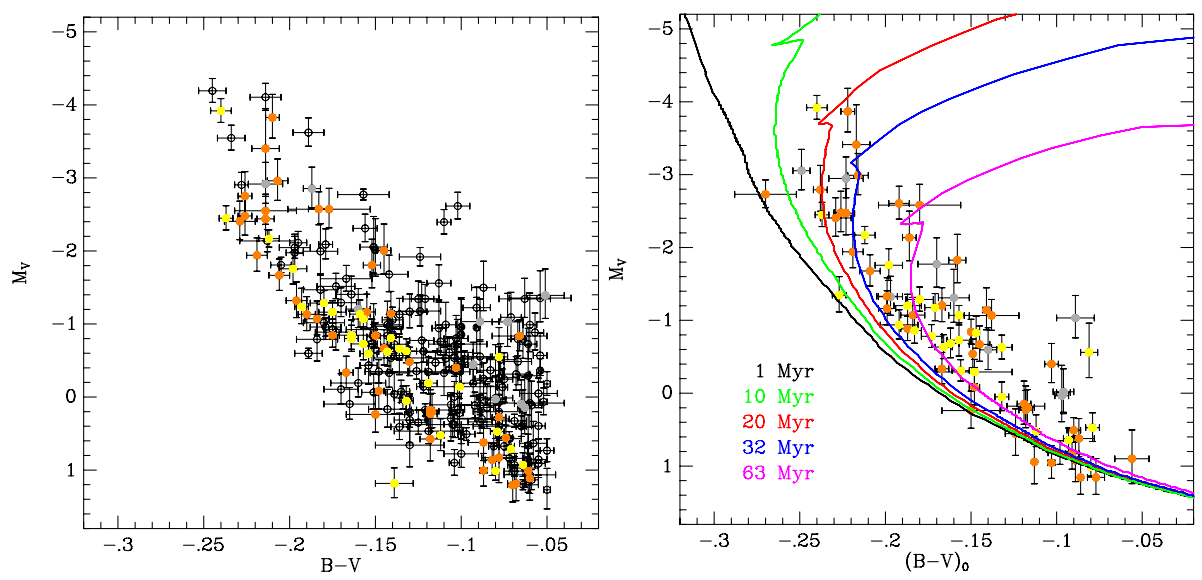

Fig. 4 Left panel: Colour-magnitude diagram of the final sample. Members of the Upper Centaurus Lupus subgroup of Sco OB2 are highlighted in orange, Lower Centaurus Crux in yellow, and Upper Scorpius in grey, respectively. Right panel: Dereddened colour-magnitude diagram of the members of the Upper Scorpius (grey), Upper Centaurus Lupus (orange) and Lower Centaurus Crux (yellow) subgroups. The solid lines are theoretical isochrones colour coded according to their ages

\section{Results and Discussion}

\subsection{The Search for the "Smoking Gun"}

The colour-magnitude diagram presented in the left panel of Fig. 4 shows a clearly discernible main sequence, which is particularly well delineated by the members of the UCL group. The turn-off point at the tip is defined by both the members of the UCL and the LCC subgroups. Apparently these together with the US subgroup are indeed the youngest OB associations in the solar neighbourhood (de Geus et al. 1989; Sartori et al. 2003). In order to determine the main sequence turnoff point we have compared the colour-magnitude diagram with theoretical isochrones calculated by Schaller et al. (1992) for solar metallicities. Fortunately de Bruijne (1999) and Sartori et al. (2003) have determined individually for 
most members of the US, UCL, and LCC subgroups, respectively, the extinction and colour excess by comparing the observed $(V-I)_{\mathrm{C}}$ colours with the intrinsic colours of stars of the same spectral type and luminosity class. Dereddened data of the 79 stars are shown together with isochrones in the right panel of Fig. 4. We conclude from Fig. 4 that the ages of the UCL and LCC subgroups lie in the range of 20 to $30 \mathrm{Myr}$, whereas our data do not allow to date the age of the US subgroup. We note that this estimate of the ages of the subgroups is nearly twice of that of de Geus et al. (1989), who determined an age of 11-12 Myr of the LCC subgroup and 14-15 Myr of the UCL subgroup, respectively. These age estimates were revised by Sartori et al. (2003) to 16-20 Myr on the basis of the then newly available Padova isochrones (Bertelli et al. 1994). The Schaller et al. (1992) isochrones, which we used, are an upgrade of the Maeder (1981a, 1981b, 1981c) isochrones by the Geneva group. Moreover we note that Sartori et al. (2003) have adopted for the majority of their stars the spectral types given in the Hipparcos catalogue, which led to a very coarse binning in the $(B-V)_{0}$ colour. Given these uncertainties we conclude that our age datings of the LCC and UCL subgroups are consistent with Sartori et al.'s result. This agrees also well with the age of Pleiades subgroup B1, which was suggested to be responsible for the origin of the LB by BB02, but is significantly larger than assumed by Maíz-Apellániz (2001), especially for the LCC subgroup. Moreover, we have examined with the help of the Simbad data base each star of the subgroups lying not on the main sequence and found that practically all these stars are either binaries or peculiar in the sense that they are variable, emission line stars etc., so that their position off the main sequence in the colour-magnitude diagram shown in Fig. 4 can be explained in our interpretation by such effects.

In Fig. 5 we trace back the positions of the UCL and LCC subgroup members over the last 30 Myr using again the epicycle equations (1). However, we have not used the individual space velocities of the stars, but adopted for each star the mass-weighted mean velocity of the combined subgroups. This avoids any unphysical spread of the spatial distribution of the stars at earlier times due to the errors of the space velocity components of the stars. We estimate that the center of the combined UCL and LCC sample is defined with an accuracy of $17 \times 17 \mathrm{pc}$ in $X, Y$. To the mean velocity of the stars we have added the solar motion $(U, V, W)_{\odot}=(10,5.3,7.2) \mathrm{km} / \mathrm{s}$ (Dehnen and Binney 1998), so that the orbits are calculated in the reference frame of the local standard of rest. Since interstellar gas has usually only small peculiar motions, the local interstellar gas, and with it the LB which is indicated in Fig. 5 by the contour line taken from Lallement et al. (2003), will basically corotate with the local standard of rest around the Galactic centre. This means that the LB is at rest in the reference frame of Fig. 5. As can been seen from Fig. 5 the path of the association has aligned itself $15 \mathrm{Myr}$ ago nearly parallel to the tangential $Y$-direction, the direction of Galactic rotation. Remnants of supernova explosions occurring during this period will have experienced very little shear due to the differential rotation of the Galaxy. The shear effect is described quantitatively by the term linearly proportional to time in the epicycle equation for $Y(t)$ (cf. (1)). The coefficient $X(0)-\frac{V(0)}{-2 B}$ defines the mean guiding centre radius of an orbit. If the spread of these radii is small, as was the case in the last $15 \mathrm{Myr}$, the shear effect of the Galactic differential rotation is minimized. In our view this might well explain why all supernovae occurring during that time have combined together to form the LB, while supernova remnants formed at earlier times have drifted away (cf. Fig. 5).

Next we compare in the right panel of Fig. 5 the position of the Upper Centaurus Lupus and Lower Centaurus Crux associations relative to the LB today and at earlier times and also with the present day LB contours in meridional sections through the bubble. From the left panel of Fig. 5 we estimate the Galactic longitude in which direction we expect the associations to approach. Choosing then the appropriate meridional section through the bubble 

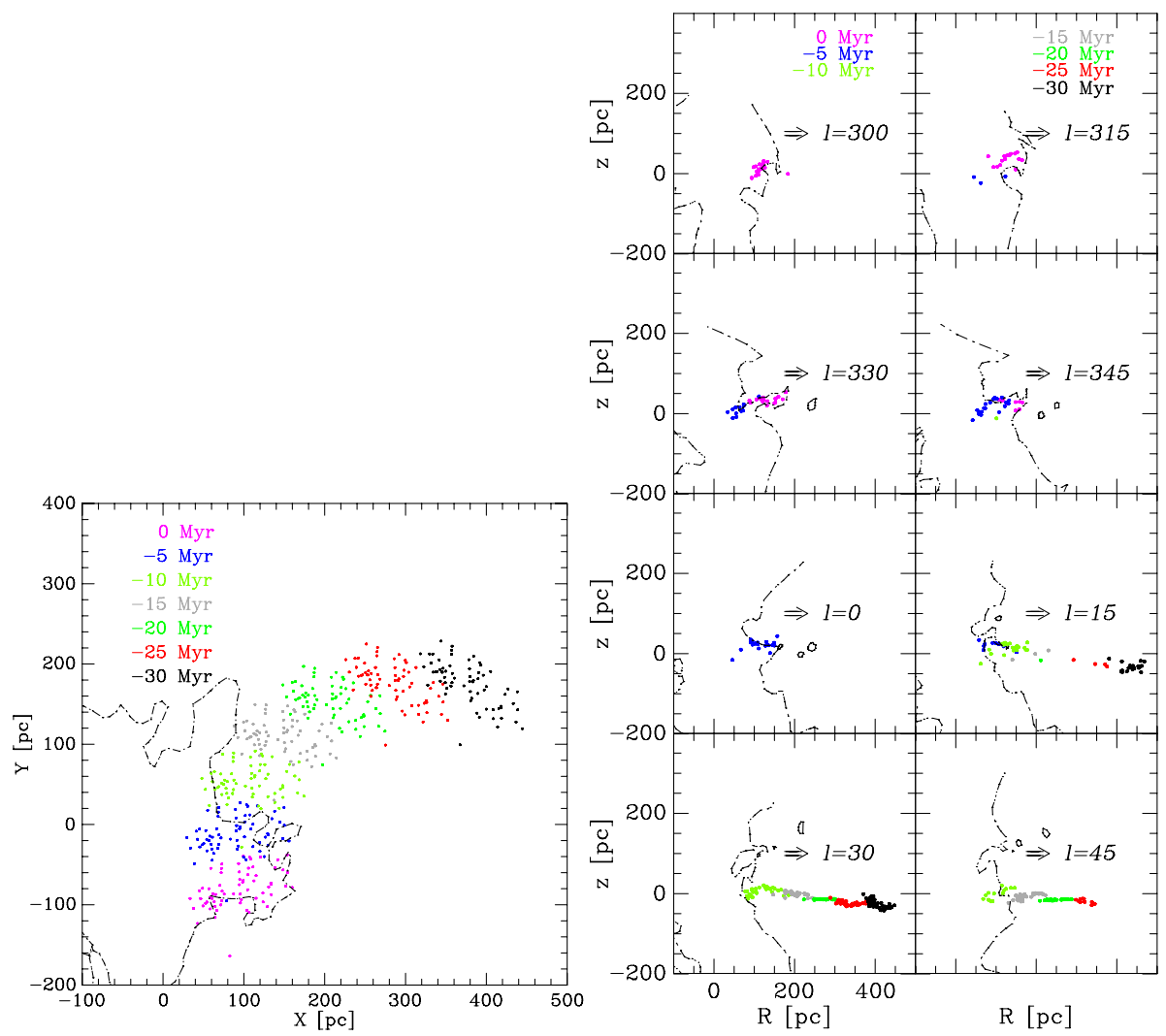

Fig. 5 Left panel: Path of the Upper Centaurus Lupus and Lower Centaurus Crux associations over the last 30 Myr projected onto the Galactic plane. The look-back time is colour coded. The orbits are calculated backwards in the reference frame of the local standard of rest assuming for each star the same mass-weighted mean velocity of the stars. The position of the Local Bubble is indicated by the dash-dotted contour line and is at rest in this reference frame. Right panel: Meridional sections of the contours delineating the outer boundary of the Local Bubble together with the positions of the stars in the Upper Centaurus Lupus and Lower Centaurus Crux associations. The horizontal axis in the upper left panel points into the direction $l=300^{\circ}$, in the upper right towards $l=315^{\circ}$ and so on. The vertical direction is always perpendicular to the Galactic midplane

from the paper by Lallement et al. (2003), we can determine immediately the positions of the stars in that longitude range relative to the LB. As can be seen from the upper right panels of Fig. 5 the associations are today just about to exit the bubble. Five and $10 \mathrm{Myr}$ ago they were inside. The bottom right panel of Fig. 5 indicates that they entered $15 \mathrm{Myr}$ ago the region occupied by the LB today. In this scenario the LB was starting to form about $15 \mathrm{Myr}$ ago, which is consistent with the estimates of the age of the LB by Maíz-Apellániz (2001) and BB02. Lallement (2007, private comm.) has announced an upgrade of the contours of the LB's outer walls based on an extended set of target stars. It will be interesting to see how the new data will support our arguments.

One might wonder how realistic a scenario is, in which the SNe explode rather close to the edge of the present day bubble. As the high resolution simulation of Fuchs et al. (2006) shows, the location of the star cluster with respect to the centre of the bubble is not 
crucial. The bubble expands always fastest in the direction of the lowest ambient density and pressure. Since in the direction of the Galactic centre the Loop I superbubble was formed almost at the same time as the LB by SNe exploding within the Sco-Cen association, the pressure in this direction is very high. Hence the LB was forced to expand rather towards the anticentre direction and perpendicular to the plane, in agreement with the observations.

\subsection{How Many Supernovae and Were They Enough to Create the LB?}

Following BB02, the number of supernovae, which have exploded during the journey of the OB association through the LB, can be estimated with the aid of the initial mass function (IMF, cf. BB02). We have fitted an IMF of the form (Massey et al. 1995).

$$
\frac{d N}{d \mathscr{M}}=\left.\frac{d N}{d \mathscr{M}}\right|_{0} \mathscr{M}^{\Gamma-1}
$$

with an index $\Gamma=-1.1 \pm 0.1$ to the data. Masses are given in units of solar masses. The lower end of the main sequence at $M_{\mathrm{V}}=1$ mag corresponds to A0 stars with masses of $\mathscr{M}_{1}=2.6 \mathscr{M}_{\odot}$ and the upper tip at $M_{\mathrm{V}}=-3.7$ mag to B0 stars with masses of $\mathscr{M}_{\mathrm{u}}=$ $8.2 \mathscr{M}_{\odot}$ (Schaller et al. 1992), respectively. The total number of stars in the UCL and LCC associations, respectively, allow the determination of the normalization constants,

$$
N=\left.\int_{2.6}^{8.2} \frac{d N}{d \mathscr{M}}\right|_{0} \mathscr{M}^{-2.1} d \mathscr{M}=\left.0.228 \frac{d N}{d \mathscr{M}}\right|_{0},
$$

implying $\left.\frac{d N}{d \mathscr{M}}\right|_{0}=184$ for the $42 \mathrm{UCL}$ and $\left.\frac{d N}{d \mathscr{M}}\right|_{0}=118$ for the 27 LCC stars, respectively. As we have shown in the previous section, OB stars entered the LB region 10-15 Myr ago, setting the clock for its origin to $t=0$. From a further fit to Schaller et al.'s isochrone data we estimate that the main sequence life time of such bright stars scales with mass as

$$
\tau=\tau_{0} \mathscr{M}^{-\alpha} \quad\left(8 \mathscr{M}_{\odot} \leq \mathscr{M} \leq 67 \mathscr{M}_{\odot}\right)
$$

with $\tau_{0}=1.6 \times 10^{8} \mathrm{yr}$ and $\alpha=0.932$. This means that the masses of the most massive stars $\mathscr{M}_{\Delta \tau}$ in the associations at a lookback time of $\Delta \tau$ years ago are given by

$$
\mathscr{M}_{\Delta \tau}=\left(\mathscr{M}_{\mathrm{u}}^{-\alpha}-\frac{\Delta \tau}{\tau_{0}}\right)^{-\frac{1}{\alpha}}
$$

implying $\mathscr{M}_{10}=15.4 \mathscr{M}_{\odot}$ if $\Delta \tau=10 \mathrm{Myr}$ or $\mathscr{M}_{15}=26.6 \mathscr{M}_{\odot}$ if $\Delta \tau=15 \mathrm{Myr}$ depending on the entry time of the associations into the volume occupied by the LB today. The expected number of supernovae, i.e. the number of 'missing' stars, is then calculated by

$$
N_{\mathrm{SN}}=\left.\int_{8.2}^{\mathscr{M}_{\Delta \tau}} \frac{d N}{d \mathscr{M}}\right|_{0} \mathscr{M}^{-2.1} d \mathscr{M} .
$$

We thus obtain estimates of $N_{\mathrm{SN}}=8-12$ from the Upper Centaurus Lupus and $N_{\mathrm{SN}}=6-8$ from the Lower Centaurus Crux associations, respectively. The estimate of 14 to 20 supernovae, which created the LB, is in good agreement with the value determined by BB02. Extrapolating the IMF to masses beyond $\mathscr{M}_{\Delta \tau}$ we estimate that 12 to $5 \mathrm{SNe}$ exploded before the associations entered the present LB volume. 
In order to assess the question, whether the estimated number of supernovae would suffice to excavate the LB, we consider the energy input by the supernova explosions into the interstellar gas. According to the initial mass function (2) there are

$$
d N=\left.\frac{d N}{d \mathscr{M}}\right|_{0} \mathscr{M}^{\Gamma-1} d \mathscr{M}
$$

stars in the mass range $(\mathscr{M}, \mathscr{M}+d \mathscr{M})$ with main sequence life times $(\tau, \tau-d \tau)$. Thus

$$
d N=\left.\frac{d N}{d \mathscr{M}}\right|_{0} \mathscr{M}^{\Gamma-1}\left(-\frac{d \mathscr{M}}{d \tau}\right) d \tau,
$$

and the energy input rate is given by (cf. BB02)

$$
\dot{\mathcal{E}}_{\mathrm{SN}}=\frac{d}{d t} \mathcal{E}_{\mathrm{SN}} N_{\mathrm{SN}}=\mathcal{E}_{\mathrm{SN}} \frac{d N_{\mathrm{SN}}}{d t}=\left.\mathcal{E}_{\mathrm{SN}} \frac{d N}{d \mathscr{M}}\right|_{0} \mathscr{M}^{\Gamma-1}(-1) \frac{d}{d \tau}\left(\frac{\tau}{\tau_{0}}\right)^{-\frac{1}{\alpha}} \frac{d \tau}{d t},
$$

where $\mathcal{E}_{\mathrm{SN}}$ denotes the energy released by a single supernova, $\mathcal{E}_{\mathrm{SN}}=10^{51}$ ergs. According to the way we have set up (8) $\frac{d \tau}{d t}$ is equal to 1 . Equation (9) describes the trade-off of the increasing number of supernova progenitors and their increasing main-sequence life times with decreasing mass. Inserting the age-to-mass relation (4) into (9) leads then to

$$
\dot{\mathcal{E}}_{\mathrm{SN}}=\dot{\mathcal{E}}_{\mathrm{SN} 0} t_{7}^{-\frac{\Gamma+\alpha}{\alpha}}
$$

with $t_{7}$ defined as $t_{7}=t / 10^{7}$ yr. For the constant $\dot{\mathcal{E}}_{\mathrm{SN} 0}$ we find

$$
\dot{\mathcal{E}}_{\mathrm{SN} 0}=\left.\frac{\mathcal{E}_{\mathrm{SN}}}{\alpha \tau_{0}} \frac{d N}{d \mathscr{M}}\right|_{0}\left(\frac{10^{7} y r}{\tau_{0}}\right)^{-0.1803}=3.5 \times\left. 10^{35} \frac{d N}{d \mathscr{M}}\right|_{0} \mathrm{erg} / \mathrm{s} .
$$

Equation (10) shows a rather weak decline of the supernova energy input rate into the LB as a result of partial compensation between the increasing number of stars with decreasing mass and a corresponding increase in main sequence life time.

Winds from hot massive stars can potentially contribute significantly to the energy input into the interstellar gas (Lamers and Cassinelli 1999). However we do not expect this for the stars which we consider here. B stars with masses below $30 \mathscr{M}_{\odot}$ have negligible main sequence winds. According to Maeder and Meynet (1987) a star with an initial mass of $20 \mathscr{M}_{\odot}$ loses then during the supergiant phase with a duration of $1.3 \mathrm{Gyrs}$ mass at a rate of about $10^{-5.5} \mathscr{M}_{\odot} \mathrm{yr}^{-1}$, or in total $5.7 \mathscr{M}_{\odot}$. Assuming as an upper limit a terminal speed of the stellar wind of $100 \mathrm{~km} / \mathrm{s}$ this implies an energy input into the ISM of $5.7 \times 10^{47} \mathrm{ergs}$ which has to be compared with the energy release by a supernova of $10^{51} \mathrm{ergs}$.

BB02 have derived a bubble wind equation which describes the growth of the size of the bubble with time. The radius of the bubble is given by

$$
R_{\mathrm{b}}(t)=R_{\mathrm{b} 0} t^{\frac{2 \alpha-\Gamma}{5 \alpha}}
$$

with the constant

$$
R_{\mathrm{b} 0}=\left[\frac{475 \alpha}{(4 \alpha-7 \Gamma)(3 \alpha-4 \Gamma)}\right]^{\frac{1}{5}} \times\left[\frac{\alpha \dot{\mathcal{E}}_{\mathrm{SN} 0} \tau_{0}^{1+\Gamma / \alpha}}{2 \pi(2-\Gamma) \rho_{0}}\right]^{\frac{1}{5}} .
$$


In (12) a constant density $\rho_{0}$ of the ambient interstellar gas is assumed for which we adopt a value of $\rho_{0}=2 \times 10^{-24} \mathrm{~g} / \mathrm{cm}^{3}$. The index in (12), $(2 \alpha-\Gamma) / 5 \alpha=0.564$, lies between the index of 0.4 of the Sedov equation, describing supernova remnants, and the index of 0.6 of the stellar wind/superbubble expansion law. For a LB age of 10 to $15 \mathrm{Myr}$ (12) predicts a bubble radius of $78 \mathrm{pc}$ to $100 \mathrm{pc}$, respectively. This in good agreement with the observed size of the LB in the Galactic disk, as determined by Lallement et al. (2003) (cf. also Fig. 5). For the determination of the expected LB size we have used the expected numbers of supernovae both from the LCC and UCL subgroups. Maíz-Apellániz (2001) has argued that the LB owes its existence only to the $6 \mathrm{SNe}$ stemming from the LCC subgroup, because stars from this subgroup came closest to the Sun in the past. We find the same when tracing the orbits of the stars backwards in time. However, the members of the UCL subgroup did enter the region occupied by the LB today and SNe stemming from the UCL subgroup have to be taken into account, in our view, in the energy considerations as well. The energy input of $6 \mathrm{SNe}$ would excavate a bubble with radius of only $65 \mathrm{pc}$, which is more difficult to reconcile with the fact that the walls of the LB have been blown out above and below the Galactic plane so that the LB has become effectively a chimney. In general, however, similarity solutions as applied here can only give a rough estimate of the LB age and size due to several severe restrictions. Firstly, the ambient medium has to be assumed to be either homogeneously distributed or to follow a power law distribution in density, and its pressure has to be small compared to the bubble pressure. Secondly, turbulent mixing and mass loading, which occur in real bubbles, are hard to incorporate without further assumptions (Dyson et al. 2002). Therefore the most realistic approach to model existing bubbles is to perform 3D high-resolution numerical simulations of their formation. A first simulation of this kind was carried out by Breitschwerdt and de Avillez (2006) which was based on the older and less detailed LB formation scenario of BB02, but which took into account the presence of the neighbouring Loop I. In Fuchs et al. (2006) we presented an upgrade of that simulation which is now based on the better understood supernova rate and the calculated paths of their progenitors through the LB. The simulations are described in detail in the contribution by Breitschwerdt et al. in this volume.

\section{Conclusions}

We have reported on a fresh, unbiased search for the OB associations in the solar vicinity which might have harboured the progenitors of the core collapse supernovae responsible for the creation of the LB. We analyzed a volume complete set of B stars in the Hipparcos catalogue and our Arivel data base. Tracing back the stars' paths in time we were able to isolate a kinematically homogenous group of stars which we identify with the $\mathrm{OB}$ association we were looking for. Following this strategy we confirm the rather robust result that besides the Upper Scorpius (US) subgroup the Upper Centaurus Lupus (UCL), and Lower Centaurus Crux (LCC) subgroups of the Sco OB2 association with ages of 20-30 Myr are the youngest stellar associations in the solar neighbourhood. However, we also found other B stars with similar kinematics. A tracing back of the stellar orbits showed that the association entered the region of the present LB 10 to $15 \mathrm{Myr}$ ago. This estimate of the LB's age is in excellent agreement with an estimate obtained by numerical simulations of the ISM during the formation of LB and Loop I triggered by supernovae in a realistic background. Deriving Ovi column densities from their simulations de Avillez and Breitschwerdt (2005), Breitschwerdt and de Avillez (2006), Fuchs et al. (2006) found that OVI absorption line data taken with FUSE (Oegerle et al. 2005; 
Savage and Lehner 2006) could be well fitted adopting a LB age of about 14 Myr. We therefore conclude that the LB must have been excavated during this time. Modelling the IMFs of the LCC and UCL subgroups we find that about 14 to $20 \mathrm{SNe}$ originated from the associations. The implied energy input into the ambient interstellar gas explains quantitatively the present size of the LB.

A future task will be to study the stars in Loop I in the same way as in the LB. We hope to develop then a self-consistent scenario of the simultaneous formation of the LB and Loop I.

Acknowledgements This research has made extensive use of the Simbad data base at CDS, Strasbourg, France. This work has been partially funded by the Portuguese Science Foundation under the project PESO/P/PRO/40149/2000 to MAdeA and DB.

\section{References}

T.W. Berghöfer, D. Breitschwerdt, Astron. Astrophys. 390, 299 (2002) (BB02)

G. Bertelli, A. Bressan, C. Chiosi, F. Fagotto, E. Nasi, Astron. Astrophys. Suppl. Ser. 106, 275 (1994)

A. Blaauw, Annu. Rev. Astron. Astrophys. 2, 213 (1964)

D. Breitschwerdt et al., Space Sci. Rev. (2008, this issue)

D. Breitschwerdt, M.A. de Avillez, Astron. Astrophys. Lett. 452, L1 (2006)

D.P. Cox, P.R. Anderson, Astrophys. J. 253, 268 (1982)

M.A. de Avillez, D. Breitschwerdt, Astrophys. J. 634, L65 (2005)

J.H.J. de Bruijne, Mon. Not. R. Astron. Soc. 310, 585 (1999)

E.J. de Geus, P.T. de Zeeuw, J. Lub, Astron. Astrophys. 216, 44 (1989)

W. Dehnen, J. Binney, Mon. Not. R. Astron. Soc. 298, 387 (1998)

P.T. de Zeeuw, R. Hoogerwerf, J.H.J. de Bruijne, A.G.A. Brown, A. Blaauw, Astron. J. 117, 354 (1999)

R. Drimmel, R.L. Smart, M.G. Lattanzi, Astron. Astrophys. 354, 67 (2000)

J.E. Dyson, S.J. Arthur, T.W. Hartquist, Astron. Astrophys. 390, 1063 (2002)

R. Egger, B. Aschenbach, Astron. Astrophys. 294, L25 (1995)

M. Feast, P. Whitelock, Mon. Not. R. Astron. Soc. 291, 683 (1997)

B. Fuchs, D. Breitschwerdt, M.A. de Avillez, C. Dettbarn, C. Flynn, Mon. Not. R. Astron. Soc. 373, 993 (2006)

J. Holmberg, C. Flynn, Mon. Not. R. Astron. Soc. 352, 440 (2004)

R. Hoogerwerf, L.A. Aguilar, Mon. Not. R. Astron. Soc. 306, 394 (1999)

D.E. Innes, T.W. Hartquist, Mon. Not. R. Astron. Soc. 209, 7 (1984)

H. Kamaya, Astron. J. 128, 761 (2004)

R. Lallement, Astron. Astrophys. 422, 391 (2004)

R. Lallement, B.Y. Welsh, J.L. Vergely, F. Crifo, D. Sfeir, Astron. Astrophys. 411, 447 (2003)

H.J.G.L.M. Lamers, J.P. Cassinelli, Introduction to Stellar Winds (Cambridge Univ. Press, Cambridge, 1999)

B. Lindblad, Handbuch der Physik, vol. 53 (1959), p. 21

A. Maeder, Astron. Astrophys. 93, 136 (1981a)

A. Maeder, Astron. Astrophys. 99, 97 (1981b)

A. Maeder, Astron. Astrophys. 102, 401 (1981c)

A. Maeder, G. Meynet, Astron. Astrophys. 182, 243 (1987)

J. Maíz-Apellániz, Astrophys. J. 560, L83 (2001)

P. Massey, K.E. Johnson, K. Degioia-Eastwood, Astrophys. J. 454, 151 (1995)

J.C. Mermilliod, B. Hauck, M. Mermilliod, The general catalogue of photometric data (2000)

W.R. Oegerle, E.B. Jenkins, R.L. Shelton, D.V. Bowen, P. Chayer, Astrophys. J. 622, 377 (2005)

M.J. Sartori, J.R.D. Lépine, W.S. Dias, Astron. Astrophys. 404, 913 (2003)

B.D. Savage, N. Lehner, Astrophys. J. Suppl. Ser. 162, 134 (2006)

G. Schaller, D. Schaerer, G. Meynet, A. Maeder, Astron. Astrophys. Suppl. Ser. 96, 269 (1992)

D.M. Sfeir, R. Lallement, F. Crifo, B.Y. Welsh, Astron. Astrophys. 346, 785 (1999)

R.K. Smith, D.P. Cox, Astrophys. J. Suppl. Ser. 134, 283 (2001) 